Www.jmscr.igmpublication.org Impact Factor 5.244

Index Copernicus Value: 5.88 ISSN (e)-2347-176x ISSN (p) 2455-0450 crossref DOI: http://dx.doi.org/10.18535/jmscr/v4i6.07

Journal Of Medical Science And Clinical Research

\title{
Comparison of Acute Toxicities in Conventional and Hypofractionated Radiotherapy in Post-Mastectomy Breast Cancer
}

(Research Article)

Authors

\section{Dr Ramesh Purohit ${ }^{1}$, Dr Neeti Sharma ${ }^{2}$, Dr Neeti Sharma ${ }^{3}$, Dr Rajesh Kumar ${ }^{4}$, Dr Shankar Lal Jakhar ${ }^{5}$}

1,3,4 $3^{\text {rd }}$ Year Pg Student, Department of Radiotherapy, ATRCTRI, SPMC, Bikaner, Rajasthan, India

${ }^{2}$ Associate Professor, Department of Radiation Oncology, ATRCTRI, SPMC, Bikaner, Rajasthan, India

${ }^{5}$ Assistant Professor, Department of Radiation Oncology, ATRCTRI, SPMC, Bikaner, Rajasthan, India

Corresponding Author

Dr Ramesh Purohit

$3^{\text {rd }}$ year PG Student, Department of Radiotherapy

ATRCTRI, SPMC, Bikaner, Rajasthan, India

\begin{abstract}
Background: Breast cancer is one of the most frequently diagnosed malignancy in women worldwide. A multimodality approach is usually preferred for treatment of all the patients for local regional and systemic control of the disease. In recent times, there has been a gradual improvement in radiotherapy delivery and has shifted from conventional to hypofractionated radiotherapy with equivocal results. This article highlights the acute toxicities in conventional and hypofractionated radiotherapy.

Aim: To study the acute toxicity in terms of skin reaction, dysphagia and arm oedema. Material and methods: This prospective study was conducted at the Department of Radiation Oncology, Acharya Tulsi Regional Cancer Treatment \& Research Institute, Bikaner. From Jan 2014 to Dec 2014; fifty patients of post-MRM carcinoma breast stage IIA-IIIA (pT1-3, pN1-2) were enrolled for the study. Informed consent patients was taken. Arm A: 50 Gy /25fractions, over 5weeks. Arm B: 40 Gy / 15 fractions, over 3 weeks. Patients were kept supine with arm abducted to 90 degrees or higher and were treated with two field technique by using Co-60 energy source on Theratron 780C or Bhabhatron-II. Toxicity was documented according to CTCAE 3.0 version. Raw or cream Aloe-vera application to the irradiated skin was advised in every patient. $P$ values of $<0.05$ were considered to be statistically significant.
\end{abstract}

Results: skin reactions observed at end of $R T$ in $68 \% \mathrm{v} / \mathrm{s} 44 \%(P=0.023)$ and at $3 \mathrm{months}, 12 \% \mathrm{v} / \mathrm{s} 4 \%$ $(P=0.045)$. Grade 2 reactions were seen in $28 \% \mathrm{v} / \mathrm{s} 8 \%(P=0.0008)$, Grade 3 reactions were also significantly lower in study arm $4 \% \mathrm{v} / \mathrm{s} 12 \%(P=0.045)$. At the end of $R T$, study arm had grade $1 \& 2$ dysphagia in $32 \% \mathrm{v} / \mathrm{s}$ $12 \%$ patients in control arm $(P=0.002)$. At 1 month dysphagia observed in $16 \%$ patients in study arm $\mathrm{v} / \mathrm{s} 4 \%$ in control arm $(P=0.007)$. No patient had grade 3 or higher dysphagia. At the end of $R T$, cosmetic appearance was equivocal. But at 1 month, cosmetic appearance was significantly better in study arm $80 \% \mathrm{v} / \mathrm{s} 52 \%$ $(P=0.014) .32 \%$ had moderate arm edema in control arm as compared to $28 \%$ in study arm $(P$ value $=0.28)$. Conclusion: Present study has proved it slightly better or equivocal with conventional fractionation for postmastectomy irradiation in terms of tolerance. Thus hypo-fractionation schedule can be adopted as a standard form of treatment in post-mastectomy patients.

Keywords: Breast cancer, Hypofractionation, Acute toxicities, Radiotherapy. 


\section{Introduction}

Breast cancer is the most frequently diagnosed cancer in India and worldwide and is the leading cause of cancer death among females. ${ }^{1,2}$ Radiotherapy (RT) is an integral part of treatment of breast cancer and indicated in selected patients after modified radical mastectomy(MRM). ${ }^{3}$ In India most of the patients undergo MRM, followed by conventional fractionation schedule which delivers a total dose of 50 Gy in 25 fractions at 2 Gy per fraction treated 5 days a week. ${ }^{4,5}$ It usually takes 6-7 weeks to complete the radiotherapy treatment by conventional fractionation. Hypofractionation is now widely practiced in post-mastectomy patients. The largest studies on hypo-fractionation in breast cancer was UK standardization of Breast Radiotherapy (START A\&B). ${ }^{6,7}$ Long term results of these trials are now available and have encouraged radiation oncologists all-over the world to adopt hypofractionation in breast cancer patients. ${ }^{8}$ In START trial B, hypo-fractionated schedule of 40 Gy in 15 fractions was delivered in 3 weeks. ${ }^{7}$ some centers in India have started practicing this hypofractionated schedule in post-mastectomy patients, the data for hypo-fractionation is lacking. In this study we aim to evaluate the acute toxicities of schedule using 15 fractions of 2.66Gy compared to conventional fractionation in post-mastectomy radiotherapy.

\section{Aim}

To study the acute toxicity in terms of skin reaction, dysphagia and arm oedema.

\section{Materials \& Methods}

This prospective study was conducted at the Department of Radiation Oncology, Acharya Tulsi Regional Cancer Treatment \& Research Institute, Bikaner. From Jan 2014 to Dec 2014; fifty patients of post-MRM carcinoma breast stage IIAIIIA (pT1-3, pN1-2) were enrolled for the study. Inclusion Criteria were patients between 18-65 years, ECOG performance score 0-2, with biopsy proven invasive carcinoma of breast that underwent MRM and received adjuvant chemotherapy as per institute protocol and referred for adjuvant radiotherapy. Exclusion Criteria were locally advanced or metastatic carcinoma breast, severely deranged liver and kidney function tests, uncontrolled co-morbidities and previously irradiated on chest wall and/or neck region. After written informed consent patients were randomized to two arms of equal strength. Arm A: 50 Gy / 25fractions, over 5weeks. Arm B: 40 Gy / 15 fractions, over 3 weeks. Patients were kept supine with arm abducted to 90 degrees or higher and were treated with two field technique by using Co-60 energy source on Theratron $780 \mathrm{C}$ or Bhabhatron-II. Chest wall was treated by medial tangential and lateral tangential beams. Supraclavicular field was treated if indicated by antero-posterior beam. To reduce the incidence of radiation pneumonitis, the lung volume treated in the tangential fields was leveled by keeping central lung distance to $\leq 3$ $\mathrm{cm}$. Weekly review was done to assess compliance, toxicity and was documented according to CTCAE 3.0 version. Treatment was prescribed to all patients for prevention and treatment of radiation induced skin toxicities according to institute protocol. Raw or cream Aloe-vera application to the irradiated skin was advised in every patient. Dietary advice and treatment was prescribed for the radiation induced dysphagia according to institute protocol. Statistical Analysis was done using SPSS 20.0 software. P values of $<0.05$ were considered to be statistically significant. $1^{\text {st }}$ follow-up of patients was after 1 month of completion of radiotherapy; subsequent follow up at 3 monthly interval.

\section{Results}

At the end of RT, 68\% patients in the control arm whereas $44 \%$ in study arm had skin reactions $(\mathrm{P}=0.023)$. At 3 months follow-up, 12\% patients in control arm whereas $4 \%$ in study arm had skin reactions $(\mathrm{P}=0.045)$. Grade 2 reactions were seen in $28 \%$ patients in control arm whereas only $8 \%$ patients in study arm $(\mathrm{P}=0.0008)$. Grade 3 
reactions were also significantly lower in study arm $4 \% \mathrm{v} / \mathrm{s} 12 \%$ in control arm $(\mathrm{P}=0.045)$. No patient in either arm had grade 4 or higher skin reactions. At the end of RT, study arm had grade $1 \& 2$ dysphagia in $32 \%$ cases as compared to $12 \%$ patients in control arm $(\mathrm{P}=0.002)$. At 1 month follow-up $16 \%$ patients in study arm had dysphagia v/s $4 \%$ in control arm $(\mathrm{P}=0.007)$. No patient had grade 3 or higher dysphagia. At the end of RT, there was non-significant difference between good to average cosmetic appearance $56 \%$ in control arm v/s $64 \%$ in study arm $(\mathrm{P}=0.46)$. But at 1 month follow-up, there was significant improvement in cosmetic appearance; $52 \%$ in control arm v/s $80 \%$ in study arm $(\mathrm{P}=0.014)$. From 3 months on this difference becomes non-significant. $32 \%$ had moderate arm edema in control arm as compared to $28 \%$ in study arm $(\mathrm{P}$ value $=0.28)$.

\section{Discussion}

In this study there was a significant difference in the occurrence of acute reactions between the two arms at the end of radiotherapy. Overall rate of skin reactions was $68 \%$ in conventional fractionation as compared to $44 \%$ in the hypofractionation arm $(\mathrm{P}$ value $=0.023)$. Grade 2 skin reaction was observed in $28 \%$ in the control arm as compared to only $8 \%$ in study arm ( $\mathrm{P}$ value $=$ 0.0008 ). Grade 3 reactions were also significantly lower in the hypo-fractionation arm. Only about 4\% patients had grade 3 reaction in the hypofractionation group with no grade 4 toxicity noted in any of the patients. At the completion of 6 months post radiotherapy no patient had a persisting erythema. Thus radiotherapy was very well tolerated by the Indian population with very less acute skin toxicity. ${ }^{9}$

In our study, aloe-vera application was advised in all patients for prevention and treatment of radiation induced skin reactions as per institute protocol. Aloe-vera application had reduced incidence and severity of skin reactions. This also led to earlier recovery of skin reactions. No patient had residual pigmentation at 6 months follow-up. In this study, there was a significantly higher incidence of dysphagia among the patients of the hypo-fractionation arm as compared to conventional arm. At the end of radiotherapy $32 \%$ patients in hypo-fractionation arm had grade 1 or 2 dysphagia v/s only $12 \%$ in the conventional fractionation. There was no grade 3 or higher dysphagia in any patient. Dysphagia began to appear in the second week onward and peaked till the end of radiotherapy. At 1 month follow-up $16 \%$ patients in the hypo-fractionation arm had persistent dysphagia whereas only $4 \%$ in conventional arm. There was no persistent swallowing difficulty beyond 3 months follow-up. The dysphagia was managed appropriately with standard treatment including dietary modifications, topical anesthetics such as viscous lidocaine, proton pump inhibitors, and promotility agents. No patient required Ryle's tube feeding. Arm edema was comparable between the two arms. There was gradual increase in number of patients with mild to moderate arm edema in both the arms. 32\% had moderate arm edema in control arm as compared to $28 \%$ in study arm ( $\mathrm{P}$ value $=$ $0.28)$.

To summarize, hypo-fractionation in breast cancer is an issue that can have widespread implications in breast cancer throughout the world. Overall hypo-fractionation has proved to be slightly better or equivocal with conventional fractionation for PMRT. This has an added advantage of reducing the overall treatment time by almost 2 weeks per patient which helps to reduce the machine load.

\section{Conclusion}

Acute skin reactions were significantly lower in the hypofractionation arm. However manageable dysphagia (grade 1\&2) appeared in significantly higher number of patients (about 1/3rd) in the hypo-fractionation arm. Pnemonitis and arm oedema were comparable between the two arms Hypo-fractionation has evolved as standard treatment of post-mastectomy. Present study has proved it slightly better or equivocal with conventional fractionation for post-mastectomy 
irradiation in terms of tolerance. Thus hypofractionation schedule can be adopted as a standard form of treatment in post-mastectomy patients.

\section{References}

1. Perez and Brady's Principles and Practice of Radiation Oncology, 6th Edition.

2. Globocan.iarc.fr. GLOBOCAN Cancer Fact Sheets: Breast cancer [Internet]. 2015 [cited 30 December 2015]. Available from: http://globocan.iarc.fr/old/FactSheets/canc ers/breast-new.asp

3. Clarke M, Collins R, Darby $S$, et al. Effects of radiotherapy and of differences in the extent of surgery for early breast cancer on local recurrence and 15-year survival: an overview of the randomised trials. Lancet. 2005.366:20872106.

4. Narendra H, Ray S. Breast conserving surgery for breast cancer: Single institutional experience from Southern India. Indian Journal of Cancer. 2011;48(4):415.

5. Overgaard M, Bentzen SM, Christensen JJ, Madsen EH: The value of the NSD formula in equation of acute and late radiation complications in normal tissue following 2 and 5 fractions per week in breast cancer patients treated with postmastectomy irradiation. Radiother Oncol 1987;9:1-11.

6. Bentzen SM, Agrawal RK, Aird EG, et al. The UK Standardization Of Breast Radiotherapy (START) Trial A of radiotherapy Hypofractionation for treatment of early breast cancer: A randomized trial. Lancet Oncol 2008; 9:331-341

7. Bentzen SM, Agrawal RK, Aird EG, et al. The UK Standardization of Breast Radiotherapy (START) Trial B of radiotherapy hypofractionation for treatment of early breast cancer: A randomized trial. Lancet 2008; 371:10981107.

8. Haviland J, Owen J, Dewar J, Agrawal R, Barrett J, Barrett-Lee $\mathrm{P}$ et al. The UK Standardisation of Breast Radiotherapy (START) trials of radiotherapy hypofractionation for treatment of early breast cancer: 10-year follow-up results of two randomised controlled trials. The Lancet Oncology. 2013;14(11):1086-1094.

9. Hijal T, Al Hamad AA, Niazi T, et al (2010). Hypofractionated radiotherapy and adjuvant chemotherapy do not increase radiation-induced dermatitis in breast cancer patients. Curr Oncol, 17, 22-7. 